\title{
Desemprego e Flexibilidade de Salários em um Contexto Evolucionário
}

\author{
Jorge Eduardo de Castro Soromenho* \\ Jaylson Jair da Silveira**
}

\begin{abstract}
Resumo
Neste artigo apresentamos um modelo de jogo evolucionário, por meio do qual discutimos as relações entre flexibilidade de salários e desemprego, em uma economia com barganhas salariais descentralizadas. As estratégias dos trabalhadores consistem em um número finito, porém arbitrariamente grande, salários nominais, e as firmas escolhem os níveis de emprego maximizadores de lucros. Mostramos que: 1) o modelo comporta múltiplos equilíbrios com homogeneidade de salários nominais (de estratégia pura) e com heterogeneidade de salários nominais (de estratégia mista); 2) o equilíbrio de médio prazo é selecionado por um processo de aprendizagem social, representado como uma dinâmica replicadora; e 3) conceber a variação dos salários nominais como um processo evolucionário, no qual os trabalhadores procuram a melhor estratégia, implica que a economia não tende necessariamente ao pleno emprego.
\end{abstract}

\section{Palavras-Chave}

modelo keynesiano, desemprego, jogos evolucionários

\section{Unemployment and Wage Flexibility in an Evolutionary Setting}

\begin{abstract}
We make use of an evolutionary game approach to study the relation between wage flexibility and unemployment in an economy with decentralized wage bargains. In our model, labor unions pick a nominal wage out of a finite set with cardinality $n \geq 2$ in each period, while firms choose employment levels that maximize their profits. We are able to show that: 1) the economy presents multiple equilibria, some are characterized by homogeneity of nominal wages (pure strategy equilibrium) and others by heterogeneity of nominal wages (mixed strategy equilibrium); 2) the medium-run equilibrium is selected by a social learning process, given by a replicator dynamics; and 3) the selected medium-run equilibrium may not be the full employment equilibrium.
\end{abstract}

\section{Keywords}

keynesian model, unemployment, evolutionary games

\section{JEL Classification}

$\mathrm{E} 12, \mathrm{C} 73$

+ Artigo recebido em outubro de 2009 e aceito para publicação em fevereiro de 2011

* Departamento de Economia FEA-USP - Endereço para contato: Av. Prof. Luciano Gualberto, 908 - Cidade Universitária - São Paulo - SP - CEP 05508-010 - Email: jecs@usp.br .

** Departamento de Ciências Econômicas - Universidade Federal de Santa Catarina - UFSC - Campus Universitário - Centro Sócio-Econômico - Trindade - Florianópolis - SC - CEP 88040-970 - Email: jaylson@cse.ufsc.br. 


\section{Introdução}

Na literatura macroeconômica keynesiana, a flexibilidade de salários nominais é crucial para garantir a autoequilibração da economia no pleno emprego. Há dois modos clássicos de conceber e formalizar a flexibilidade. ${ }^{1}$ Primeiro, se o salário se ajusta instantaneamente ao excesso de demanda, diz-se que ele é perfeitamente flexível. Neste caso, ele é variável endógena, e o pleno emprego é o único equilíbrio possível. Segundo, se o salário nominal, embora rígido no curto prazo, muda em decorrência do excesso de demanda ao longo do tempo, caracteriza-se a flexibilidade imperfeita. Em geral, o segundo caso é formalizado por meio de equações diferenciais ou a diferenças finitas, cuja especificação assegura que a taxa de variação do salário apresenta o mesmo sinal do excesso de demanda no mercado de trabalho. Assim, a própria definição do processo de tâtonnement garante que a única trajetória estacionária é a de pleno emprego. Evidentemente, a flexibilidade imperfeita, ao contrário da primeira, permite a investigação de propriedades dinâmicas interessantes, notadamente em decorrência dos efeitos riqueza (Pigou - Patinkin - Haberler), Keynes e Mundell. ${ }^{2}$ É possível, então, que o equilíbrio de pleno emprego seja instável; não obstante, ele ainda é o único admissível - e, em verdade, na maior parte dos modelos dinâmicos, o equilíbrio clássico é um atrator local. Em suma, as duas formas consagradas de discutir a flexibilidade de salários proporcionam, de modo geral, o mesmo resultado: na ausência de rigidez nominal, as economias capitalistas, ao contrário do que propôs o próprio Keynes, equilibram-se no pleno emprego.

A flexibilidade de salários pode, alternativamente, ser examinada num ambiente de jogos evolucionários (SOROMENHO \& SILVEIRA, 2008). Sob essa perspectiva, variações dos salários nominais não mais resultam do processo quase mecânico (KOOPMANS, 1957) descrito pela equação de tâtonnement, mas sim das escolhas de diferentes alternativas ou estratégias por parte dos trabalhadores. Em Soromenho \& Silveira (2008), as duas estratégias disponíveis para os trabalhadores são exigir um específico salário rígido ou optar pela perfeita flexibilidade. A segunda estratégia garante o emprego a todos os que aceitam o salário flexível. Obtém-se, então, um modelo no qual existem dois setores, keynesiano e clássico. A segunda estratégia, embora útil para os propósitos daquele artigo, traz consigo a premissa de que há algum processo de barganha - não modelado explicitamente -, que se desenrola a uma velocidade relativamente alta (tecnicamente infinita) e assegura que o salário nominal acordado iguale o salário real à produtividade marginal no nível de pleno emprego do setor clássico.

1 Ver, por exemplo, Hahn \& Solow (1997) e Tobin (1993 e 1994).

2 Ver Sargent, (1987) e, principalmente, Flaschel, Frenke \& Semmler (1994, capítulos II e III). O trabalho clássico nessa linha de investigação é de Tobin (1975); o mesmo autor apresenta em dois artigos uma apreciação ampla sobre a longa controvérsia a respeito da estabilidade no modelo keynesiano (TOBIN, 1993 e 1994). 
Neste trabalho adotamos um enfoque diferente. Para discutir as relações entre flexibilidade salarial e desemprego, supomos que as estratégias dos trabalhadores consistem em um número finito, porém arbitrariamente grande, de salários nominais. Descartamos, portanto, a presença de um setor clássico nos moldes delineados acima. Não obstante, o modelo deve, evidentemente, comportar o pleno emprego como possibilidade. Assim, dados os parâmetros, em especial o dispêndio autônomo, um dos salários nominais possíveis deve ser tal que, se todos os trabalhadores da economia optarem por ele, vigora a taxa natural de desemprego.

A escolha das estratégias é um processo descentralizado - como é típico de uma economia de mercado -, no qual diferentes salários podem, em princípio, coexistir num mesmo instante do tempo. Cabe, então, ao próprio processo dinâmico microfundamentado selecionar os equilíbrios. A discussão das relações entre salários nominais e desemprego passa a ser concebida como uma ecologia de regras e a questão é saber se - e sob que circunstâncias - é possível garantir que o equilíbrio de pleno emprego é um atrator.

O artigo está organizado da seguinte forma: na seção 2 é apresentado o modelo básico estático de curto prazo; na seção 3, formalizamos o processo de escolha das estratégias ou salários nominais que ocorrem na transição entre períodos; apresentase na seção 4 o sistema dinâmico de médio prazo que resulta desse processo; são discutidos, na seção 5, os múltiplos equilíbrios que o médio prazo comporta - especificamente, provamos a existência de equilíbrios de estratégia pura e mista; na seção 6, demonstramos que todos os equilíbrios de estratégia pura, os quais correspondem a diferentes níveis de emprego, são atratores locais e que, portanto, a economia não tende, em geral, à plena ocupação da mão-de-obra; na seção 7 apresentamos uma simulação que ilustra a coevolução dos salários reais, dos níveis de emprego total e dos níveis de emprego associados a cada estratégia; encerramos o artigo com a seção 8, apresentando as conclusões e uma breve interpretação de Keynes à luz do modelo proposto.

\section{O Modelo Básico}

Seja uma economia na qual é produzido um único tipo de bem por $h$ firmas. No início de período, em cada firma e de forma descentralizada, empresários e colegiados de trabalhadores negociam salários nominais. Supomos o seguinte arranjo institucional: em cada empresa, os trabalhadores (ou colegiados) impõem, no início de período, um específico salário nominal, mas não podem assegurar o salário real e a manutenção de seus empregos, que resultam do funcionamento da economia como um todo. Os critérios que presidem suas escolhas serão discutidos mais adian- 
te. Formalmente, definimos $s \geq 2$ estratégias indicadas pelos salários nominais $w_{i}$, $i=1, \ldots s$. Firmas e trabalhadores podem, então, ser agrupados em setores indexados segundo a estratégia adotada pelos colegiados.

No curto prazo, a produção de uma firma típica ou representativa do setor $i$ é uma função Cobb-Douglas do número de trabalhadores, $L_{i}$, e do estoque de capital, $K$, que supomos constante e igual para as firmas de todos os setores:

$$
f\left(L_{i}, K\right)=L_{i}^{1-\beta} K^{\beta}, \quad 0<\beta<1
$$

As firmas maximizam lucro ao igualarem a produtividade marginal do trabalho ao salário real, o que permite obter as demandas de trabalho e as ofertas de produto das firmas típicas dos vários setores:

$$
\begin{aligned}
& L_{i}\left(p, w_{i}, K\right)=K\left[\frac{(1-\beta) p}{w_{i}}\right]^{1 / \beta} \\
& f\left(L_{i}\left(p, w_{i}, K\right), K\right)=K\left[\frac{(1-\beta) p}{w_{i}}\right]^{(1-\beta) / \beta}
\end{aligned}
$$

Sejam: $N$ o total de trabalhadores; $N_{i}$ o número de trabalhadores do setor $i ; U$ o total de desempregados; e $h_{i}$ o número de empresas nas quais vigora a estratégia $i$. Considere as seguintes definições: $n_{i}=N_{i} / N \geq 0 ; u=U / N \geq 0$; e $\alpha_{i}=h_{i} / h \geq 0$. Evidentemente, essa economia deve atender às restrições naturais:

$$
\sum_{i=1}^{s} \alpha_{i}=1 ; \quad u+\sum_{i=1}^{s} n_{i}=1
$$

O total de trabalhadores no setor $i$ é $N_{i}=h_{i} L_{i}$. Normalizando por $N$, temos $n_{i}=\alpha_{i} L_{i} / c$, onde $c$ é a relação entre os totais de trabalhadores e de firmas da economia, $N / h$. Assim, a demanda de trabalho normalizada do setor $i$ é:

$$
n_{i}=\frac{\alpha_{i} K}{c}\left[\frac{(1-\beta) p}{w_{i}}\right]^{1 / \beta}
$$


A oferta de produto de cada setor é $h_{i} f($.). Dividindo pelo número total de firmas, $h$, definimos a oferta normalizada por $y_{i}=\alpha_{i} f($.$) . Logo, Somando a produção de$

$$
y_{i}=\alpha_{i} K\left[\frac{(1-\beta) p}{w_{i}}\right]^{(1-\beta) / \beta}
$$

todos os setores, obtemos a oferta agregada (igualmente normalizada por $h$ ):

$$
\sum_{i=1}^{s} y_{i}=K(1-\beta)^{\frac{1-\beta}{\beta}} p^{\frac{1-\beta}{\beta}} \sum_{i=1}^{s} \alpha_{i} w_{i}^{-\frac{1-\beta}{\beta}}
$$

Completamos o modelo de curto prazo com uma demanda agregada, igualmente normalizada pelo número total de firmas, que é função do nível de preços e de um parâmetro $A>0$ :

$$
D(A, p)=\frac{A}{p}
$$

A função pode ser interpretada como resultado da substituição de uma $L M$ numa $I S$. A dependência em relação ao nível de preços é uma forma sintética de incorporar os efeitos Keynes e riqueza; o parâmetro $A$ capta os demais fatores que afetam a demanda (gasto autônomo, estoque de moeda etc).

O equilíbrio no mercado de produto requer, portanto, que:

$$
\frac{A}{p}=K(1-\beta)^{\frac{1-\beta}{\beta}} p^{\frac{1-\beta}{\beta}} B
$$

onde $B=\sum_{i=1}^{s} \alpha_{i} w_{i}^{-(1-\beta) / \beta}>0$.

Isolando $p$ em (9) obtemos o preço de equilíbrio: ${ }^{3}$

$$
p^{*}(\alpha, A)=\frac{A^{\beta}}{K^{\beta}(1-\beta)^{1-\beta} B^{\beta}}
$$

sendo $\alpha=\left(\alpha_{1}, \ldots \alpha_{s}\right)$ o vetor de distribuição setorial das firmas. Substituindo (10) em (5), identificamos os níveis setoriais de emprego:

3 Por economia de notação, designaremos $p^{*}(\alpha, A)$ simplesmente por $p^{*}$ sempre que inexistir ambiguidade. O mesmo procedimento será adotado para as demais funções. 


$$
n_{i}^{*}(\alpha, A)=\frac{(1-\beta) A \alpha_{i} w_{i}^{-\frac{1}{\beta}}}{c B}, \quad i=1, \ldots S
$$

Seja $\Delta_{s}=\left\{\alpha \in R_{+}^{s}: \sum_{i=1}^{s} \alpha_{i}=1\right\}$. As soluções (10) e (11), que definem o equilíbrio de curto prazo e atendem à restrição natural das firmas, são funções

$p^{*}: \Delta_{s} \times R_{++} \rightarrow R_{++}$e $n_{i}^{*}: \Delta_{s} \times R_{++} \rightarrow R_{+}$. Definimos o nível de emprego total e a taxa de desemprego por: ${ }^{4}$

$$
\begin{aligned}
& \eta^{*}(\alpha, A)=\sum_{i=1}^{s} n_{i}^{*}=\frac{A(1-\beta) \sum_{i=1}^{s} \alpha_{i} w_{i}^{-\frac{1}{\beta}}}{c B} \\
& u^{*}(\alpha, A)=1-\eta^{*}(\alpha, A)
\end{aligned}
$$

No curto prazo, o nível de preços e as taxas de emprego por setor respondem a variações do componente autônomo da demanda agregada de modo usual:

$$
\begin{aligned}
& \frac{\partial p^{*}}{\partial A}=\frac{\beta A^{-(1-\beta)}}{K^{\beta}(1-\beta)^{1-\beta} B^{\beta}}>0 \\
& \frac{\partial n_{i}^{*}}{\partial A}=\frac{(1-\beta) \alpha_{i} w_{i}^{-\frac{1}{\beta}}}{c B}>0, \quad i=1, \ldots S
\end{aligned}
$$

\section{A Escolha das Estratégias}

Considere o tempo como uma variável contínua. A taxa de variação do número de empresas de um setor $i$ pode ser expressa como a diferença entre o influxo (sobrescrito $I$ ) e o efluxo (sobrescrito $E$ ) das firmas para esse setor:

$$
\dot{h}_{i}=\dot{h}_{i}^{I}-\dot{h}_{i}^{E} .
$$

Como o total de firmas é constante, dividindo ambos os lados por $h$ obtemos:

4 Observe que, para atender à restrição natural dos trabalhadores, é necessário impor uma condição adicional a respeito do componente autônomo da demanda, a saber, $A$ deve ser tal que $\eta^{*} \leq 1$. 


$$
\dot{\alpha}_{i}=\frac{\dot{h}_{i}^{I}-\dot{h}_{i}^{E}}{h}
$$

O colegiado de trabalhadores de uma empresa do setor $i$ compara seu resultado com o de outra empresa $j$ escolhida aleatoriamente segundo uma distribuição uniforme. Assim, a probabilidade de um colegiado de trabalhadores do tipo $i$ comparar seu payoff com um colegiado do tipo $j$ é dada por:

$$
P(i, j)=\frac{h_{j}}{h} \geq 0
$$

tal que $\sum_{j=1}^{s} P(i, j)=1$.

$\mathrm{Na}$ comparação de resultados, supomos que os colegiados de trabalhadores levam em consideração os salários reais e a probabilidade de assegurar o emprego, que consideramos igual à taxa de desemprego efetiva vigente - para o setor $i$, por exemplo, essa taxa é $n_{i} /\left(n_{i}+u\right)$. Definimos, então,

$$
\omega_{i} \equiv \frac{w_{i}}{p} \frac{n_{i}}{n_{i}+u} ; \omega_{j} \equiv \frac{w_{j}}{p} \frac{n_{j}}{n_{j}+u}
$$

Os resultados têm, portanto, o estatuto de salários reais esperados. Supomos que a mudança de estratégia só se efetiva caso a diferença de salários reais esperados, obtida pela comparação em pares, seja superior a uma diferença mínima $\boldsymbol{E}$, que definimos com uma variável aleatória com função densidade de probabilidade uniforme com suporte $[-a, a]$, onde $a=\max \left\{\left|\omega_{i}-\omega_{j}\right|\right\}, \forall i \forall j, i \neq j \cdot{ }^{5}$ Logo, a probabilidade de um agente revisor mudar de estratégia é:

$$
F(z)=P(\varepsilon<z)=\int_{-a}^{z} \frac{1}{2 a} d \varepsilon=\frac{a+z}{2 a}
$$

5 A variável aleatória $\varepsilon$ pode ser vista como a diferença entre duas variáveis aleatórias $\varepsilon_{i \text { e }} \varepsilon_{j}$ associadas aos colegiados de trabalhadores dos tipos $i$ e $j$, respectivamente. Como salienta Weibull (1995, p. 157), essas variáveis podem ser interpretadas como erros ou ruídos de observação dos agentes ou ainda como diferenças idiossincráticas entre as preferências dos agentes. Nachbar (1990) sugere uma outra interpretação para $\mathcal{E}$, a saber, um custo de mudança de estratégia, não estabelecido de maneira determinista e pago instantaneamente no momento da efetivação da mudança. Qualquer dessas três formas de interpretar a variável aleatória $\boldsymbol{E}$ seria apropriada para o caso em análise. 
onde $z$ é a diferença de resultados.

Assim, um revisor $i$ adota a estratégia $j$ se: (1) compara seu resultado com o de $j$, sendo $P(i, j)$ a probabilidade de isso ocorrer; (2) se $z=\omega_{j}-\omega_{i}>\varepsilon$, cuja probabilidade é dada por (20). Logo, substituindo (19) em (20) e multiplicando por $P(i, j)$, obtemos a probabilidade de um revisor $i$ migrar para a estratégia $j$ :

$$
i \rightarrow j: \frac{h_{j}}{h} \frac{1}{2 a}\left(a+\omega_{j}-\omega_{i}\right), \quad j=1, \ldots . n ; j \neq i
$$

Procedendo de modo análogo para o caso de um revisor $j$, identificamos a probabilidade dele adotar a estratégia $i$ :

$$
j \rightarrow i: \frac{h_{i}}{h} \frac{1}{2 a}\left(a+\omega_{i}-\omega_{j}\right), \quad j=1, \ldots n ; j \neq i .
$$

Supondo que todos os colegiados de trabalhadores avaliam suas escolhas simultaneamente e uma única vez a cada unidade de tempo, o total de revisores efetivos do tipo $i$ que migram para a estratégia $j$ é obtido multiplicando a probabilidade (21) por $h_{i}$. Somando sobre $j$, identificamos o efluxo da população de firmas $i$ :

$$
\dot{h}_{i}^{E}=\sum_{j \neq i}^{s} h_{i} \frac{1}{2 a} \frac{h_{j}}{h}\left(a+\omega_{j}-\omega_{i}\right)
$$

Efetuando a mesma operação para os agentes das demais estratégias $j$, obtemos o influxo para $i$ :

$$
\dot{h}_{i}^{I}=\sum_{j \neq i}^{s} h_{j} \frac{1}{2 a} \frac{h_{i}}{h}\left(a+\omega_{i}-\omega_{j}\right)
$$

Ao substituirmos as duas últimas equações em (17), temos a taxa de variação das empresas da população $i: 6$

$$
\dot{\alpha}_{i}=\frac{\alpha_{i}}{a} \sum_{j=1}^{s} \alpha_{j}\left(\omega_{i}-\omega_{j}\right) \text {. }
$$

6 A inclusão do elemento $\alpha_{j}\left(\omega_{j}-\omega_{j}\right)$ no somatório simplesmente facilita a notação. 


\section{O Sistema Dinâmico}

A equação (25) permite identificar os fluxos para a estratégia $i$. Com $s$ estratégias, temos o seguinte sistema dinâmico, que incorpora as soluções do curto prazo:

$$
\dot{\alpha}_{i}=\frac{\alpha_{i}}{a} \sum_{j=1}^{s} \alpha_{j} \phi^{i, j}(\alpha, A) ; \quad i=1, \ldots, s
$$

onde

$$
\phi^{i, j}(\alpha, A)=\omega_{i}(\alpha, A)-\omega_{j}(\alpha, A)=\frac{1}{p^{*}}\left(\frac{w_{i} n_{i}^{*}}{n_{i}^{*}+u^{*}}-\frac{w_{j} n_{j}^{*}}{n_{j}^{*}+u^{*}}\right) .
$$

Como $\sum \dot{\alpha}_{i}=0$, há $s-1$ equações independentes.

O espaço de estados do sistema é o conjunto $\Delta_{s}=\left\{\alpha \in R_{+}^{s}: \sum_{i=1}^{s} \alpha_{i}=1\right\}$, ou seja, o simplex unitário de dimensão $s-1$, que é um subconjunto não vazio, compacto e convexo de $R^{s}$. Denotamos por $e^{i}, i=1, \ldots s$, os vértices do simplex, i. e., os vetores cuja coordenada $i$ é igual a um e as demais iguais a zero. Cada vértice é dito uma estratégia pura.

Indicamos por $\operatorname{int}\left(\Delta_{s}\right)=\left\{\alpha \in \Delta_{s}: \alpha_{i}>0, \forall i\right\}$ o interior (relativo ao hiperplano $\left\{\alpha \in R^{s}: \sum_{i=1}^{s} \alpha_{i}=1\right\}$ ) do simplex; e por $\partial\left(\Delta_{s}\right)=\left\{\alpha \in \Delta_{s}: \alpha_{i} \notin \operatorname{int}\left(\Delta_{s}\right)\right\}$ a borda ou fronteira do simplex. Assim, um vetor pertencente à borda possui pelo menos uma coordenada igual a zero.

Suponha que nossa atenção esteja focada no comportamento do sistema no caso em que mantemos algumas estratégias permanentemente zeradas. Seja $L$ o conjunto das estratégias remanescentes. Indicamos por $\Delta_{\# L} \subset \Delta_{s}$ o fecho convexo das estratégias puras pertencentes a $L$ e por $\operatorname{int}\left(\Delta_{\# L}\right)$ e $\partial\left(\Delta_{\# L}\right)$ o interior (relativo) e a borda de $\Delta_{\# L}$, respectivamente. Observe que: (a) um vetor pertencente a $\operatorname{int}\left(\Delta_{\# L}\right), \# L<s$ pertence, por definição, a $\partial\left(\Delta_{s}\right)$; e (b) se $\alpha_{i} \notin L$, então, $\alpha_{i}=0$.

Antes de analisarmos os equilíbrios e suas propriedades, é necessário discutir dois pontos: primeiro, identificar como o nível de emprego total $\eta^{*}$ se comporta no simplex; segundo, examinar sob que hipóteses as relações $\phi^{i, j}$ são funções continuamente diferenciáveis em $\Delta_{s}$, para podermos utilizar os instrumentos usuais da análise dinâmica. 
(i) Variação do nível de emprego total no espaço de estados.

Inicialmente, considere as derivadas da função emprego total definida em (12):

$$
\frac{\partial \eta^{*}}{\partial \alpha_{j}}=\frac{A(1-\beta)}{c B^{2}}\left(w_{j}^{-\frac{1}{\beta}} B-w_{j}^{-\frac{1-\beta}{\beta}} \sum_{i=1}^{s} \alpha_{i} w_{i}^{-\frac{1}{\beta}}\right), \quad j=1, \ldots s
$$

A função $\eta^{*}$ e suas derivadas são bem definidas e contínuas para todo $\alpha$ pertencente a um conjunto aberto contido em $R^{s}$, tal que $B \neq 0$. Porém $\Delta_{s}$ é fechado e, em geral, não podemos definir derivadas parciais em conjuntos fechados. Logo, para discutirmos o comportamento de $\eta^{*}$ no simplex, é conveniente estender as funções e suas derivadas sobre um superconjunto aberto de $\Delta_{s}$. Nesse sentido, considere o seguinte hiperplano formado pelos vetores $\alpha$ para os quais $B$ é igual a zero e, portanto, $\eta^{*}$ e suas derivadas são indeterminadas:

$$
H_{B}=\left\{\alpha \in R^{s}: \sum_{i=1}^{s} \alpha_{i} w_{i}^{-(1-\beta) / \beta}=0\right\}
$$

Os conjuntos $\Delta_{s}$ e $H_{B}$ são fechados e disjuntos, pois $\sum_{i=1}^{s} \alpha_{i} w_{i}^{-(1-\beta) / \beta}>0$ para qualquer $\alpha \in \Delta_{s}$. Logo, ${ }^{7}$ existem conjuntos abertos $X_{1} \supset \Delta_{s}$ e $X_{2} \supset H_{B}$ tais que $X_{1} \cap X_{2}=\varnothing$ e podemos estender $\eta^{*}$ e suas derivadas sobre $X_{1}$. Observe que a mesma extensão pode ser feita para as funções ${ }^{8} u^{*}$ e $n_{i}^{*}$, pois $u^{*}=1-\eta^{*}$ e $\eta^{*}=\sum n_{i}^{*}$.

Admita, agora, sem perda de generalidade, que $w_{1}$ é o menor salário nominal. Considere um vetor $\alpha \in \Delta_{s}$ e uma redução do número de empresas nas quais é adotada a estratégia $s=1$. Consequentemente aumenta o número de empresas nas quais vigoram estratégias alternativas, ou seja, o sistema desloca-se para algum vetor $\bar{\alpha} \in \Delta_{s}$ para o qual $\bar{\alpha}_{1}<\alpha_{1}, \bar{\alpha}_{j} \geq \alpha_{j}, \forall j \neq 1$ e $\sum_{j=2}^{s}\left(\bar{\alpha}_{j}-\alpha_{j}\right)=\alpha_{1} \bar{\alpha}_{1}$.

A partir do vetor aplicado $\bar{\alpha}-\alpha$, construímos o vetor unitário $\hat{\alpha}=(\bar{\alpha}-\alpha) /\|\bar{\alpha}-\alpha\|$. A variação do nível total de emprego decorrente de uma alteração de $\alpha_{1}$ na direção do vetor $\hat{\boldsymbol{\alpha}}$ é dada, então, pela derivada direcional: ${ }^{9}$

$$
D \eta_{\alpha}^{*}(\hat{\alpha})=\nabla \eta^{*} \cdot \hat{\alpha}
$$

7 Ver, por exemplo, Lipschutz (1965: 115, teorema 8.8).

8 Para não sobrecarregar a notação, utilizamos os mesmos símbolos $\eta^{*}$ e $n_{i}^{*}$ para as funções estendidas.

9 Note que, em decorrência da extensão, as derivadas parciais do gradiente são contínuas em $X_{1}$. 
Considerando a definição de $\hat{\boldsymbol{\alpha}}$, temos,

$$
\hat{\alpha}\|\bar{\alpha}-\alpha\|=\left(-\sum_{j=2}^{s}\left(\bar{\alpha}_{j}-\alpha_{j}\right), \bar{\alpha}_{2}-\alpha_{2}, \ldots, \bar{\alpha}_{s}-\alpha_{s}\right),
$$

o que permite reescrever a derivada direcional como:

$$
D \eta_{\alpha}^{*}(\hat{\alpha})=\left(\frac{\partial \eta^{*}}{\partial \alpha_{2}}-\frac{\partial \eta^{*}}{\partial \alpha_{1}}\right) \frac{\left(\bar{\alpha}_{2}-\alpha_{2}\right)}{\|\bar{\alpha}-\alpha\|}+\ldots+\left(\frac{\partial \eta^{*}}{\partial \alpha_{s}}-\frac{\partial \eta^{*}}{\partial \alpha_{1}}\right) \frac{\left(\bar{\alpha}_{s}-\alpha_{s}\right)}{\|\bar{\alpha}-\alpha\|}
$$

Observe que $\left(\bar{\alpha}_{j}-\alpha_{j}\right) /\|\bar{\alpha}-\alpha\|$ é positivo para $j=2, \ldots s$ e estritamente positivo para pelo menos um $j$. O sinal de cada termo não nulo de (32) é igual, portanto, ao sinal de $\left(\partial \eta^{*} / \partial \alpha_{j}-\partial \eta^{*} / \partial \alpha_{1}\right)$. Recordando (28), temos que:

$$
\frac{\partial \eta^{*}}{\partial \alpha_{j}}-\frac{\partial \eta^{*}}{\partial \alpha_{1}}=\frac{A(1-\beta)}{c B^{2}}\left[\left(w_{j}^{-\frac{1}{\beta}}-w_{1}^{-\frac{1}{\beta}}\right) B-\left(w_{j}^{-\frac{1-\beta}{\beta}}-w_{1}^{-\frac{1-\beta}{\beta}}\right) \sum_{i=1}^{s} \alpha_{i} w_{i}^{-\frac{1}{\beta}}\right.
$$

Substituindo $B$ e simplificando, a expressão entre colchetes fica:

$$
\sum_{i=1}^{s}\left[\alpha_{i} w_{i}^{-\frac{1}{\beta}}\left(\left(w_{j}^{-\frac{1}{\beta}}-w_{1}^{-\frac{1}{\beta}}\right) w_{i}-\left(w_{j}^{-\frac{1-\beta}{\beta}}-w_{1}^{-\frac{1-\beta}{\beta}}\right)\right)\right]
$$

Como $\alpha_{i} w_{i}^{-1 / \beta} \geq 0$, valendo a desigualdade estrita para pelo menos um $i$, uma forma simples de identificar o sinal de (33) é interpretar:

$$
\left(w_{j}^{-\frac{1}{\beta}}-w_{1}^{-\frac{1}{\beta}}\right) w_{i}-\left(w_{j}^{-\frac{1-\beta}{\beta}}-w_{1}^{-\frac{1-\beta}{\beta}}\right)
$$

como uma função de $w_{i}$. Dado que $-\left(w_{j}^{-(1-\beta) / \beta}-w_{1}^{-(1-\beta) / \beta}\right)$ é positivo e $\left(w_{j}^{-1 / \beta}-w_{1}^{-1 / \beta}\right)$ é negativo, pois $w_{1}<w_{j}$ para todo $j=2, \ldots, s$, a função é estritamente decrescente com relação a $w_{i}$. Portanto, o seu valor máximo ocorre em $w_{i}=w_{1}$ e é igual a $w_{j}^{-\frac{1}{\beta}}\left(w_{1}-w_{j}\right)<0$. Logo, (35) é negativo para todo $i$. Por conseguinte, $\partial \eta^{*} / \partial \alpha_{j}-\partial \eta^{*} / \partial \alpha_{1}<0$ e o sinal da derivada direcional $D \eta_{\alpha}^{*}(\hat{\alpha})$ é negativo. 
Concluímos, então, que uma redução do número de empresas nas quais é adotada a estratégia de menor salário nominal diminui o emprego. Segue-se que o maior nível de ocupação da mão-de-obra ocorre em $e^{1}$.

A Figura 1 (a) ilustra esses resultados para o caso em que $s=3$. Nela, um deslocamento a partir de $\alpha$ em direção a qualquer vetor situado na região cinza implica redução do nível de emprego; na direção do vetor tracejado, a redução de $\alpha_{1}$ resulta exclusivamente em aumento de $\alpha_{3}$; na direção do vetor cheio preto, o aumento dar-se-á apenas em $\alpha_{2}$.

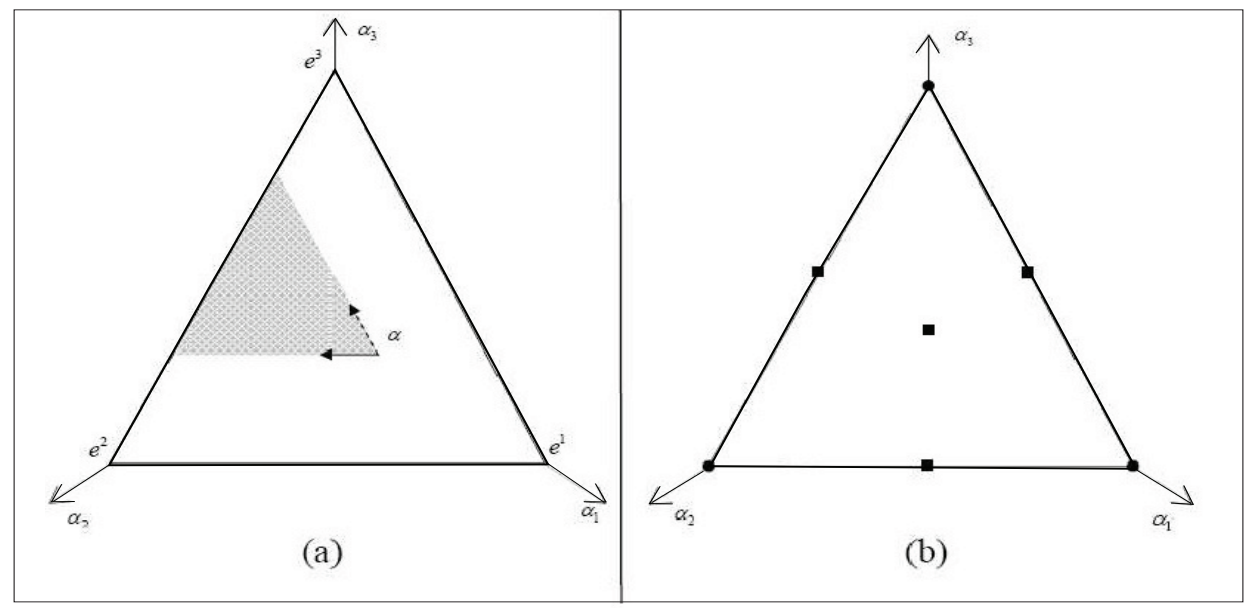

(ii) As funções $\phi^{i, j}(\alpha, A)$.

A discussão apresentada neste item compreende dois pontos: (1) garantir a definição $\phi^{i, j}$ para todo $\alpha$ pertencente ao simplex; e (2) estender essa função e suas derivadas para um conjunto aberto.

Como as relações $\phi^{i, j}=\omega_{l}-\omega_{j}$ resultam de operações elementares que envolvem as funções $p^{*}, n_{i}^{*}$ e $u^{*}$, as quais são definidas sobre $\Delta_{s} \times R_{++}$, a diferença de salários reais esperados será função definida sobre o mesmo domínio caso essas operações não resultem em indeterminações. Cada salário real esperado, $\omega_{v}$, possui como denominador a expressão $p^{*}\left(n_{v}^{*}+u^{*}\right)$. A função $p^{*}$ assume valores estritamente positivos; todavia, $n_{v}^{*} \in[0,1]$. Assim, se $\alpha$ e $A$ são tais que $n_{v}^{*}$ é igual a zero e inexiste desemprego, $u^{*}=0$, o salário real esperado $\omega_{v}=w_{v} n_{v}^{*} / p^{*}\left(n_{v}^{*}+u^{*}\right)$, será uma indeterminação matemática que convém evitar. $\mathrm{O}$ modo mais simples de fazê-lo é 
assegurar $u^{*}>0$, o que pode ser feito com base numa hipótese específica a respeito de $A$.

Nesse sentido, observe que sabemos da análise precedente que o maior nível de emprego ocorre em $e^{1}$. Assuma $\alpha_{1}=n_{1}=1$ na definição de $n_{i}^{*}(\alpha, A)$ apresentada em (11). Resolvendo (11) para $A$, resulta $A=c w_{1} /(1-\beta)$, ou seja, só para esse específico valor do componente autônomo há pleno emprego quando todas as empresas estiverem no setor um. ${ }^{10} \operatorname{Logo}$, se $A \leq c w_{1} /(1-\beta)$, a indeterminação de salários reais esperados só pode ocorrer em $e^{1}$. Podemos supor, então, que $A \leq \bar{n} c w_{1} /(1-\beta)$, sendo $\bar{n} \in(0,1)$ e tão próximo de um quanto queiramos. Substituindo $A=\bar{n} c w_{1} /(1-\mathrm{b})$ e $\alpha_{1}=1$ em (11), obtemos $n_{1}=\bar{n}<1$. Consequentemente, assumindo esse limite superior para $A$, temos que $u^{*}$ é estri-

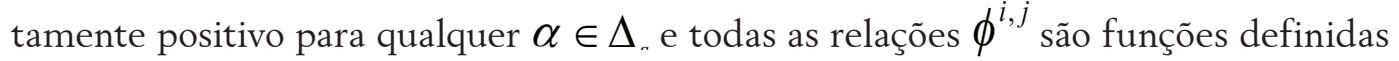
sobre $\Delta_{s} \times\left\{A \in R_{++}: A \leq \bar{n} c w_{1} /(1-\beta)\right\}$. Se quisermos dar uma interpretação econômica a uma hipótese que é apenas uma conveniência matemática, podemos definir $\bar{u}=1-\bar{n}>0$ como a taxa de desemprego natural.

Por último, vamos estender as funções $\phi^{i, j}$ e suas derivadas a um conjunto aberto. Recorde a definição dessas funções em (27). O mínimo múltiplo comum da diferença de salários reais esperados é $\left(n_{i}^{*}+u^{*}\right)\left(n_{j}^{*}+u^{*}\right) p^{*}$. As derivadas parciais de $\phi^{i, j}$ em relação a $\alpha$ terão, então, por denominador, o quadrado dessa expressão e envolverão as derivadas parciais das funções $u^{*}, n_{i}^{*}$ e $p^{*}$. Sabemos que: (1) a existência das funções $u^{*}, n_{i}^{*}$ e $p^{*}$ e de suas derivadas em relação a $\alpha$ exigem $B \neq 0$; (2) $p^{*}=A^{\beta} /\left(K^{\beta}(1-\beta)^{1-\beta} B^{\beta}\right) \neq 0$. Portanto, a indeterminação de $\phi^{i, j}$ e de suas derivadas ocorre quando $\left(n_{i}^{*}+u^{*}\right)\left(n_{j}^{*}+u^{*}\right)=0$ ou $B=0$.

Recorde $H_{B}$ definido em (29)e forme os seguintes conjuntos $H_{i}=\left\{\alpha \in R^{s}: n_{i}^{*}+u^{*}=0\right\}$, $i=1, \ldots, s$ e $H=\cup_{i=1}^{s} H_{i} \cup H_{B}$. O conjunto $H$ é fechado, pois é união finita de con juntos fechados. Com a restrição $A \leq \bar{n} c w_{1} /(1-\beta)$, a taxa de desemprego $u^{*}$ é estritamente positiva para qualquer $\alpha \in \Delta_{s}$. Logo, nenhum $\alpha$ pertencente ao simplex atende à definição dos conjuntos $H_{i}$; ademais, como assinalado anteriormente, conjuntos $\Delta_{s}$ e $H_{B}$ são disjuntos. Portanto, $H \cap \Delta_{s}=\varnothing$. Segue-se que, pelo mesmo

10 Se o parâmetro $A$ for maior que desse valor crítico, o espaço de estados relevante de um ponto de vista econômico é um subconjunto próprio de $\Delta_{S}$ e torna-se necessário definir uma fronteira de pleno emprego, como foi feito em Soromenho e Silveira (2008). A hipótese que será explicitada mais adiante evita esse inconveniente. 
teorema da separação utilizado anteriormente, existem conjuntos abertos e disjuntos $X_{3} \supset \Delta_{s}$ e $X_{4} \supset H$. As funções $\phi^{i, j}$ podem, então, ser estendidas para o aberto $X_{3}$ e são continuamente diferenciáveis para todo $\alpha \in \Delta_{s}$.

\section{Os Equilíbrios de Estratégia Pura e Mista}

Considere o sistema (26) e o conjunto de vértices do simplex, $E E P=\left\{e^{i}: i=1,2, \ldots, s\right\}$ : Proposição 1. Todo $\alpha \in E E P$ é um equilíbrio do sistema dinâmico (26).

Prova. Pela hipótese $A \leq \bar{n} c w_{1} /(1-\beta)$, as funções $\phi^{i, j}$ estão definidas para qualquer $e^{i}$. Logo, substituindo esses vetores no sistema e recordando que $\phi^{i, i}$ é sempre igual a zero, temos $\dot{\alpha}_{i}=(1 / a) \sum_{j \neq i}^{s} 0 \phi^{i, j}=0$ e $\dot{\alpha}_{v}=(0 / a) \sum_{j \neq i}^{s} \alpha_{j} \dot{\phi}^{j}=0, \forall v \neq i$.

Os elementos de $E E P$ são denominados equilíbrios de estratégia pura. Além deles, o sistema (26) apresenta outras soluções estacionárias, ditas equilíbrios de estratégia mista. Para identificá-los, observe, inicialmente, que o sistema pode ser expresso como:

$$
\begin{aligned}
\dot{\alpha}_{i} & =\frac{\alpha_{i}}{a} \sum_{j=1}^{s} \alpha_{j}\left(\omega_{i}-\omega_{j}\right)=\frac{\alpha_{i}}{a}\left(\sum_{j=1}^{s} \alpha_{j} \omega_{i}-\sum_{j=1}^{s} \alpha_{j} \omega_{j}\right)= \\
& =\frac{\alpha_{i}}{a}\left(\omega_{i}-\sum_{j=1}^{s} \alpha_{j} \omega_{j}\right) ; \quad i=1, \ldots, s .
\end{aligned}
$$

Logo, se $\omega_{i}=\sum_{j=1}^{s} \alpha_{j} \omega_{j}$ para todo $i$ - ou seja, se todos os salários forem iguais -, então $\dot{\alpha}_{i}=0$. Assim, qualquer $\alpha$ tal que $\phi^{i, j}=0, i, j=1, . . s$ é um equilíbrio estacionário do sistema dinâmico. Cumpre, então, provar a existência desses equilíbrios, o que exige alguns resultados preliminares.

\section{(i) Preliminares.}

Inicialmente, note que nem todas as funções $\phi^{i, j}$ são independentes. Para discutir este ponto, é conveniente organizar essas funções na forma de uma matriz: 


$$
m_{0}=\left[\begin{array}{ccccc}
0 & \phi^{1,2} & \ldots & \phi^{1, s-1} & \phi^{1, s} \\
\phi^{2,1} & 0 & \ldots & \phi^{2, s-1} & \phi^{2, s} \\
\vdots & \vdots & \ddots & \vdots & \vdots \\
\phi^{s-1,1} & \phi^{s-1,2} & \ldots & 0 & \phi^{s-1, s} \\
\phi^{s, 1} & \phi^{s, 2} & \ldots & \phi^{s, s-1} & 0
\end{array}\right]
$$

Como $\phi^{i, j}=\omega_{l}-\omega_{j}$ é igual a $-\phi^{j, i}=-\left(\omega_{j}-\omega_{i}\right)$, a matriz é antissimétrica. Portanto, se os elementos acima da diagonal principal forem iguais a zero, o mesmo ocorre com os demais e, então, $\dot{\alpha}_{i}=0$. Considere, agora, o conjunto formado pelos elementos imediatamente acima da diagonal principal: $\left\{\phi^{1,2}, \phi^{2,3}, \ldots, \phi^{s-1, s}\right\}$. Qualquer elemento acima da diagonal principal da matriz ou pertence a esse conjunto ou pode ser obtido por meio da soma de elementos do conjunto. Por exemplo, $\phi^{2,5}$, que não pertence a esse conjunto, pode ser obtido a partir de soma de $\phi^{2,3}+\phi^{3,4}+\phi^{4,5}$. Em termos gerais, qualquer $\phi^{i, j}$ que não pertence ao conjunto é igual a $\phi^{i, i+1}+\ldots+\phi^{j-1, j}$. Assim, o conjunto é composto de funções de diferença de salários reais esperados que permitem obter todas as demais. Segue-se que as soluções do sistema:

$$
\phi^{i, i+1}=0, i=1, \ldots s-1 \text {, }
$$

formado a partir dos elementos do conjunto, são equilíbrios de estratégia mista do sistema dinâmico com $s$ estratégias.

Suponha que uma ou mais estratégias não estão sendo adotadas (por exemplo, admita $\alpha_{2}=\alpha_{s-1}=0$ ). No sistema dinâmico (26), as equações e os elementos dos somatórios que envolvem essas estratégias são obviamente iguais a zero; no exemplo, temos:

$$
\begin{gathered}
\dot{\alpha}_{i}=\frac{\alpha_{i}}{a}\left(\alpha_{1} \phi^{i, 1}+0 \phi^{i, 2}+\sum_{j=3}^{s-2} \alpha_{j} \phi^{i, j}+0 \phi^{i, 2-1}+\alpha_{s} \phi^{i, j}\right), \quad i=1,3, \ldots, s-2, s \\
\dot{\alpha}_{i}=\frac{0}{a} \sum_{j=1}^{s} \alpha_{j} \dot{\phi}^{, j}=0, \quad i=2, s-1 .
\end{gathered}
$$

Elimine-se então do sistema $\phi^{i, j}=0, i, j=1, . . s$, as equações $\dot{\alpha}_{i}$ e as funções $\phi^{i, j}$ que são iguais a zero por hipótese. Se o sistema remanescente, que denominamos 
de sistema reduzido, possuir solução, então, $\dot{\alpha}=0$. Observe que essas soluções, caso existam, não pertencem a $\operatorname{int}\left(\Delta_{s}\right)$ - pois, por construção, supomos que alguns $\alpha_{i}$ são iguais a zero -, mas pertencem ao interior de um simplex $\Delta_{\# L}$, onde recordamos que $L$ é o conjunto das estratégias remanescentes (no exemplo, $L=\{1,3, \ldots, s-2, s\})$. Obviamente, o argumento aplica-se para os casos em que há mais de uma estratégia, $\# L \geq 2$.

No caso dos sistemas reduzidos, podemos adotar procedimento análogo ao que utilizamos no do sistema completo. Forme a matriz antissimétrica composta das funções remanescentes. No exemplo:

$$
m_{2, s-1}=\left[\begin{array}{ccccc}
0 & \phi^{1,3} & \ldots & \phi^{1, s-2} & \phi^{1, s} \\
\phi^{3,1} & 0 & \ldots & \phi^{3, s-2} & \phi^{3, s} \\
\vdots & \vdots & \ddots & \vdots & \vdots \\
\phi^{s-2,1} & \phi^{s-2,3} & \ldots & 0 & \phi^{s-2, s} \\
\phi^{s, 1} & \phi^{s, 3} & \ldots & \phi^{s, s-2} & 0
\end{array}\right] .
$$

A matriz $m_{2, s-1}$ é obtida a partir de $m_{0}$ eliminando-se as linhas e colunas 2 e $s-1 .^{11}$ Considere o conjunto dos elementos acima da diagonal principal $\left(\left\{\phi^{1,3}, \phi^{3,4}, \ldots, \phi^{s-2, s}\right\}\right.$, no exemplo). Novamente, qualquer elemento acima da diagonal principal ou é elemento do conjunto ou é igual à soma de elementos desse conjunto. ${ }^{12}$ Assim, as soluções do sistema obtido ao igualarmos os elementos acima da diagonal principal a zero são equilíbrios de estratégia mista do sistema dinâmico reduzido; e, como os $\alpha_{i}$ das demais estratégias são iguais a zero, $\dot{\alpha}=0$.

Para provar a existência dos equilíbrios de estratégia mista, é conveniente recorrer a um argumento de ponto fixo. Como se sabe, as provas de existência de equilíbrio em economia que utilizam teoremas de ponto fixo normalmente se beneficiam da lei de Walras. Para os nossos sistemas, podemos identificar uma propriedade que desempenha um papel semelhante ao que essa lei cumpre nas provas mencionadas. Com efeito, ao somarmos os elementos imediatamente acima da diagonal principal e o último elemento da primeira coluna, o resultado é sempre igual a zero. Por exemplo: (a) para o sistema completo, a soma de $\phi^{i, i+1}, i=1, \ldots S-1$, é igual a

11 O subscrito lista as estratégias eliminadas; se ele for igual a 0 , significa que todas as $s$ estratégias estão em vigor.

12 Por exemplo, $\phi^{1, s}=\phi^{1,3}+\stackrel{3,4}{\phi} \ldots+{ }_{\phi}^{s-3, s-2}+s_{\phi}^{-2, s}$. 
$\omega_{1}-\omega_{s}$; adicionando $\phi^{s, 1}$, obtemos $\omega_{1}-\omega_{s}+\omega_{s}-\omega_{1}=0$, ou seja, $\phi^{s, 1}+\sum_{i=1}^{s-1} \phi^{i, i+1} \equiv 0$; (b) para a matriz $m_{2, s-1}$, temos $\phi^{s, 1}+\phi^{1,3}+\sum_{i=3}^{s-3} \phi^{i, i+1}+\phi^{s-2, s} \equiv 0$.

No intuito de proporcionar um tratamento uniforme para todos os sistemas, seja $L(m)$ o conjunto ordenado (segundo a ordem natural) das estratégias associadas a uma matriz $m$. Note que o número de conjuntos possíveis depende do valor de $s$. Por exemplo, para $s=3$ temos, além $L\left(m_{0}\right)$, mais três conjuntos formados a partir das combinações duas a duas das três estratégias, $C(3,2)$. Em termos gerais, o número de conjuntos $L(m)$ é $\sum_{x=2}^{s} C(s, x)$.

Um elemento de $L(m)$ é, portanto, uma estratégia $i$. Definimos por $k(i)$ o sucessor imediato de $i$, com a convenção de que se $i$ for o último elemento de $L(m)$, então $k(i)$ é o primeiro elemento do conjunto. Assim, para o sistema completo, $m$ é igual a $m_{0}$ e $L\left(m_{0}\right)=\{1,2, . . s\}$ e $k(s)=1$; para o sistema reduzido do exemplo, temos $L\left(m_{2, s-1}\right)=\{1,3, \ldots, s-2, s\}, k(1)=3, k(s-2)=s$ e $k(s)=1$. Segue-se que conhecendo os conjuntos $L(m)$ e, portanto, as matrizes $m$, as funções $\phi^{i, k(i)}$ correspondem aos elementos imediatamente acima da diagonal principal e ao último elemento da primeira coluna de $m$. Os resultados que provamos acima podem ser expressos, então, nos seguintes lemas:

Lema 1. $\sum_{i \in L(m)} \phi^{i, k(i)} \equiv 0$, para cada conjunto de estratégias $L(m), \# L(m) \geq 2$.

Lema 2. Se $\phi^{i, k(i)}=0$, para todo $i \in L(m)$, então, $\phi^{i, j}=0, \forall i \forall j$, logo, $\dot{\alpha}=0$

\section{(ii) Prova de existência dos equilíbrios de estratégia mista.}

As proposições a seguir completam a descrição dos equilíbrios do modelo. ${ }^{13}$

Proposição 2. O sistema (26) possui (pelo menos) um equilíbrio $\alpha^{*} \in \Delta_{\# L}$, tal que $\phi^{i, k(i)}\left(\alpha^{*}\right)=0, \forall i \in L(m)$, para cada conjunto de estratégias $L(m), \# L(m) \geq 2$.

Prova. A prova é feita em duas partes: (a) construímos uma função de $\Delta_{\# L}$ em $\Delta_{\# L}$ e provamos que possui um ponto fixo; (b) mostramos que a existência desse ponto fixo implica que $\phi^{i, k(i)}\left(\alpha^{*}\right)=0, \forall i \in L(m)$, o que, pelo lema 2 implica que $\alpha^{*}$ é um equilíbrio do sistema dinâmico.

13 A proposição nada mais é do que uma adaptação das conhecidas provas de ponto fixo de existência de equilíbrio geral. Ver, por exemplo, Hildenbrand \& Kirman (1988). 
(a) Seja a função $\alpha \mapsto \Omega(\alpha), \alpha \in \Delta_{\# L}$, onde:

$$
\Omega^{i}(\alpha)=\frac{\alpha_{i}+\max \left(0,-\phi^{i, k(i)}(\alpha)\right)}{1+\sum_{j \in L(m)} \max \left(0,-\phi^{j, k(j)}(\alpha)\right)}, i \in L(m) .
$$

Sabemos que $\Delta_{\# L}$ é um subconjunto não vazio, compacto e convexo de $R^{s}$. A função $\alpha \mapsto \Omega(\alpha)$, é contínua em $\Delta_{\# L}$ com valores em $\Delta_{\# L}$, pois $\sum_{i \in L(m)} \Omega^{i}(\mathrm{a})=1$, e $\Omega^{i}(\mathrm{a}) \geq 0$. Segue-se, pelo teorema de ponto fixo de Brouwer, ${ }^{14}$ que existe um ponto fixo para $\Omega$, i.e., $\Omega\left(\alpha^{*}\right)=\alpha^{*}$.

(b) A existência do ponto fixo $\alpha^{*}$ implica $\phi^{i, k(i)}\left(\alpha^{*}\right)=0$, para todo $i \in L(m)$. Com efeito, suponha o contrário, ou seja, que existe algum $i$ para o qual $\phi^{i, k(i)} \neq 0$. Há dois casos a examinar:

Primeiro, considere $\phi^{i, k(i)}\left(\alpha^{*}\right)>0$.

Temos: (i) $\alpha_{i}^{*}+\max \left(0,-\phi^{i, k(i)}\left(\alpha^{*}\right)\right)=\stackrel{*}{\alpha} \leq 1$; e (ii), como $\# L \geq 2$, existe, pelo Lema 1 , um $j$ tal que $\phi^{j, k(j)}\left(\alpha^{*}\right)<0$, e, portanto, $1+\sum_{j \in L(m)} \max \left(0,-\phi^{j, k(j)}\right)>1$. Logo, $\phi^{i, k(i)}\left(\alpha^{*}\right)>0$. implica:

$$
\Omega^{i}\left(\alpha^{*}\right)=\frac{\alpha_{i}^{*}}{1+\sum_{j \in L(m)} \max \left(0,-\phi^{j, k(j)}(\alpha)\right)}<\alpha_{i}^{*},
$$

o que contradiz que $\alpha^{*}$ é ponto fixo. Segundo, admita $\phi^{j, k(j)}\left(\alpha^{*}\right)<0$.

Segue que: (i) $1+\sum_{j \in L(m)} \max \left(0,-\phi^{j, k(j)}\right)>1$; e (ii) pelo Lema 1, existe pelo menos uma estratégia $v$ para a qual $\phi^{i, k(i)}\left(\alpha^{*}\right)>0$. o que implica $\alpha_{v}^{*}>0$ (pois, se $\alpha_{v}^{*}=0$, então, $\left.\phi^{v, k(v)} \leq 0\right)$ e, portanto, $\alpha_{v}^{*}+\max \left(0,-\phi^{v, k(v)}(\stackrel{*}{\alpha})\right)=\stackrel{*}{\alpha} \cdot \operatorname{Logo}, \phi^{j, k(j)}\left(\alpha^{*}\right)<0$ implica:

$$
\Omega^{v}\left(\alpha^{*}\right)=\frac{\alpha_{v}^{*}}{1+\sum_{j \in L(m)} \max \left(0,-\phi^{j, k(j)}(\alpha)\right)}<\alpha_{v}^{*},
$$

14 Ver, por exemplo, Hildenbrand \& Kirman (1988, appendix IV.) 
e, novamente, $\alpha^{*}$ não pode ser ponto fixo. Assim, a existência do ponto fixo implica $\phi^{j, k(j)}\left(\alpha^{*}\right)<0$ para todo $i \in L(m)$. Segue-se, pelo Lema 2 , que $\alpha^{*}$ é um equilíbrio do sistema dinâmico. A próxima proposição apresenta um resultado que, embora óbvio, convém registrar.

Proposição 3. O equilíbrio $\alpha^{*}$ pertence ao $\operatorname{int}\left(\Delta_{\# L}\right)$, onde $\alpha^{*}$ é o ponto fixo da proposição anterior.

Prova. Suponha o contrário, i.e., que o ponto fixo $\alpha^{*}$ pertence à borda do simplex, $\partial\left(\Delta_{\# L}\right)$. Logo, por definição, existe algum par de estratégias $(v, j)$ tal que $\alpha_{v}^{*}>0$ e $\alpha_{j}^{*}=0$. Mas, por um lado, $\left(\alpha_{v}^{*}>0\right) \Rightarrow\left(n_{v}^{*}>0\right)$, e, portanto, $\omega_{v}>0$; e, por outro, $\left(\alpha_{j}^{*}=0\right) \Rightarrow\left(n_{j}^{*}=0\right)$, e, consequentemente, $\omega_{v}>0$. Logo, $\phi^{v, j}\left(\alpha^{*}\right)=\omega_{v}>0$ o que contradiz a hipótese de que $\alpha^{*}$ é o ponto fixo da proposição (parte b).

Para melhor compreensão das proposições, considere o caso $s=3$ retratado na Figura 1 (b). Para $\# L=3$, a proposição 2 garante a existência de pelo menos um ponto fixo em $\Delta_{3}$; a proposição 3 assegura que esse equilíbrio situa-se em $\operatorname{int}\left(\Delta_{3}\right)$. Para $L=\{1,2\}$, as proposições asseguram a existência de equilíbrio no interior do fecho convexo de $e^{1}$ e $e^{2}$, que designamos por co $\left(e^{1}, e^{2}\right)$; para $L$ igual $\{1,3\}$ e $\{2,3\}$, os equilíbrios situam-se nos interiores de $c o\left(e^{1}, e^{3}\right)$ e $c o\left(e^{2}, e^{3}\right)$. Esses equilíbrios estão representados por quadrados na figura. Finalmente, a proposição 1 assegura a existência dos equilíbrios de estratégia pura indicados pelas bolas pretas da figura.

\section{A Estabilidade dos Equilíbrios de Estratégia Pura}

O sistema dinâmico (26) possui propriedades que permitem estabelecer que os equilíbrios de estratégia pura são atratores locais. Substituindo $\alpha_{s}$ por $1-\sum_{i=1}^{s-1} \alpha_{i}$, e considerando apenas as $s-1$ equações diferenciais independentes, o sistema pode ser reescrito em termos das $\alpha_{1}, \ldots, \alpha_{s-1}$ variáveis:

$$
\dot{\alpha}_{i}=\frac{\alpha_{i}}{a}\left(\sum_{j=1}^{s-1} \alpha_{j} \phi^{i, j}\left(\alpha_{1}, \ldots \alpha_{s-1}\right)+\left(1-\sum_{j=1}^{s-1} \alpha_{j}\right) \phi^{i, s}\left(\alpha_{1}, \ldots \alpha_{s-1}\right)\right) ; \quad i=1, \ldots, s-1
$$


A matriz jacobiana da linearização do sistema é:

$$
\left[\begin{array}{ccc}
\frac{\partial \dot{\alpha}_{1}}{\partial \alpha_{1}} & \cdots & \frac{\partial \dot{\alpha}_{1}}{\partial \alpha_{s-1}} \\
\vdots & \ddots & \vdots \\
\frac{\partial \dot{\alpha}_{s-1}}{\partial \alpha_{1}} & \cdots & \frac{\partial \dot{\alpha}_{s-1}}{\partial \alpha_{s-1}}
\end{array}\right]
$$

Recordando que $\phi^{i, j}$ é sempre igual a zero, as derivadas são:

$$
\begin{gathered}
\frac{\partial \dot{\alpha}_{i}}{\partial \alpha_{i}}=\frac{1}{a}\left(\sum_{j=1}^{s-1} \alpha_{j} \phi^{i, j}+\left(1-\sum_{j=1}^{s-1} \alpha_{j}\right) \phi^{i, s}\right)+ \\
\frac{\alpha_{i}}{a}\left(\sum_{j=1}^{s-1} \alpha_{j} \frac{\partial \phi^{i, j}}{\partial \alpha_{i}}+\left(1-\sum_{j=1}^{s-1} \alpha_{j}\right) \frac{\partial \phi^{i, s}}{\partial \alpha_{i}}-\phi^{i, s}\right) ; \quad i=1, \ldots, s-1 ; \\
\frac{\alpha_{i}\left(\sum_{j=1}^{s-1} \alpha_{j} \frac{\partial \phi^{i, j}}{\partial \alpha_{v}}+\phi^{i, v}+\left(1-\sum_{j=1}^{s-1} \alpha_{j}\right) \frac{\partial \phi^{i, s}}{\partial \alpha_{v}}-\phi^{i, s}\right) ;}{i=1, \ldots, s-1 ;} \begin{array}{l}
i=1, \ldots, s-1 ; \\
v=1 . \\
v \neq i .
\end{array}
\end{gathered}
$$

O primeiro bloco de derivadas nos dá os elementos da diagonal principal da matriz jacobiana; o segundo bloco fornece os demais elementos.

\section{(i) Elementos fora da diagonal principal.}

Considere os equilíbrios $e^{\ell}, \ell=1, \ldots, s$, isto é, os vetores para o qual $\alpha_{\ell}=1$ e todos os demais elementos são nulos. Temos dois casos a serem examinados: $\ell$ igual ou diferente de $i$. Se $\ell \neq i$, então, $\alpha_{i}=0$ e as derivadas (48) são nulas. Se $\ell=i$, então, $\ell<s$, pois $i<s$. Logo, $\alpha_{\ell}$ é elemento dos somatórios, o que implica $\sum_{j=1}^{s-1} \alpha_{j}=1 \mathrm{e}$ $\sum_{j=1}^{s-1} \alpha_{j} \partial \phi^{\ell, j} / \partial \alpha_{v}=\partial \phi^{\ell, \ell} / \partial \alpha_{v}=0$, pois se trata da derivada de uma função constante. Sobra $(1 / a)\left(\phi^{\ell, v}-\phi^{\ell, s}\right)=-\omega_{v}^{*}+\omega_{s}^{*}$ que também é igual a zero, pois, $\ell=i \neq v$ e $\alpha_{\ell}=1$ implicam $n_{v}=0$ e $n_{s}=0$, devido a (11), os quais, por sua vez, implicam $\omega_{v}^{*}$ e $\omega_{s}^{*}$ nulos, pela própria definição de salário esperado. Ou seja, todos os elementos fora da diagonal principal são zeros. 


\section{(i) Elementos da diagonal principal.}

Considere, agora, os elementos da diagonal principal (47) avaliados nos $s$ equilíbrios de estratégia pura $e^{\ell}$. Há, portanto, $s \times(s-1)$ derivadas a serem avaliadas. Novamente, devemos examinar as possibilidades $\ell$ igual ou diferente de $i$.

Se $\ell \neq i$, temos $\dot{\alpha}_{i}=0$ e o segundo termo do lado direito de (47) é nulo. As derivadas ficam reduzidas a:

$$
\left.\frac{\partial \dot{\alpha}_{i}}{\partial \alpha_{i}}\right|_{e^{\ell}}=\frac{1}{a} \sum_{j=1}^{s-1} \alpha_{j} \phi^{i, j}+\frac{1}{a}\left(1-\sum_{j=1}^{s-1} \alpha_{j}\right) \phi^{i, s}, \quad i=1, \ldots, s-1, \quad \ell=1, \ldots, s, \ell \neq i .
$$

Temos dois casos: $\ell$ igual ou diferente de $s$ (i.e. a estratégia cuja equação diferencial foi eliminada). Se $\ell \neq s$, então, $\alpha_{\ell}$ é elemento dos somatórios. Logo: (a) $\sum_{j=1}^{s-1} \alpha_{i}=1$, e o segundo termo do lado direito de (49) é igual a zero; e (b) o primeiro termo é igual a $(1 / a) \phi^{i, \ell}=(1 / a)\left(\omega_{i}-\omega_{\ell}\right)=-(1 / a) \omega_{\ell}$, pois, $\alpha_{i}=0$ implica $n_{i}=0$, e, por conseguinte, $\omega_{i}$ é igual a zero. Assim, o valor da derivada para o caso $\ell \neq s$ é $-\omega_{\ell} / a$. Se $\ell=s$, então, $\alpha_{j}=0$, para todo $j=1, \ldots, s-1$. Logo, o primeiro termo é nulo e o segundo é igual a $(1 / a) \phi^{i, \ell}=(1 / a)\left(\omega_{l}-\omega_{\ell}\right)=-(1 / a) \omega_{\ell}$.

Em suma, para $\ell \neq i$ :

$$
\left.\frac{\partial \dot{\alpha}_{i}}{\partial \alpha_{i}}\right|_{e^{\ell}}=-\frac{\omega_{\ell}}{a} ; \quad i=1, \ldots, s-1 ; \ell=1, \ldots, s ; \text { e } i \neq \ell .
$$

Obtivemos, portanto, os valores de $(s-1)(s-2)+(s-1)=(s-1)^{2}$ derivadas. Resta, então, apenas uma derivada a ser avaliada para cada um dos $e^{\ell}, \ell=1, \ldots s-1$, equilíbrios de estratégia pura, a saber, os casos para os quais $i=\ell$ (e, portanto, $\ell<s)$ :

$$
\begin{aligned}
& \left.\frac{\partial \dot{\alpha}_{\ell}}{\partial \alpha_{\ell}}\right|_{e^{\ell}}=\frac{1}{a}\left(\sum_{j=1}^{s-1} \alpha_{j} \phi^{\ell, j}+\left(1-\sum_{j=1}^{s-1} \alpha_{j}\right) \phi^{\ell, s}\right)+ \\
& \frac{1}{a}\left(\sum_{j=1}^{s-1} \alpha_{j} \frac{\partial \phi^{\ell, j}}{\partial \alpha_{\ell}}+\left(1-\sum_{j=1}^{s-1} \alpha_{j}\right) \frac{\partial \phi^{\ell, s}}{\partial \alpha_{\ell}}-\phi^{\ell, s}\right), \quad \ell=1, \ldots, s-1
\end{aligned}
$$


Como $\ell<s$, os somatórios incluem o elemento $\alpha_{\ell}=1$. Portanto: (a) $\sum_{j=1}^{s-1} \alpha_{j}=1$; (b) $\sum_{j=1}^{s-1} \alpha_{j} \phi^{\ell, j}=\phi^{\ell, \ell}=0$; e, (c) $\sum_{j=1}^{s-1} \alpha_{j} \partial \phi^{\ell, j} / \partial \alpha_{\ell}=(1 / a) \partial \phi^{\ell, \ell} \partial \alpha_{\ell}=0$. Por conseguinte, $\left.\left(\partial \dot{\alpha}_{\ell} / \partial \alpha_{\ell}\right)\right|_{e^{\ell}}=-(1 / a) \phi^{\ell, s}$. Como $\alpha_{\ell}=1 \Rightarrow \alpha_{s}=0$, que, por sua vez, implica $\omega_{s}^{*}=0$, obtemos:

$$
\left.\frac{\partial \dot{\alpha}_{\ell}}{\partial \alpha_{\ell}}\right|_{e^{\ell}}=-\frac{\omega_{\ell}}{a}, \quad \ell=1, \ldots, s-1
$$

O exame das duas possibilidades, $\ell \neq i$ e $\ell=i$, permite concluir, portanto, que os valores da diagonal principal avaliados nos equilíbrios de estratégia pura são todos iguais a um real negativo. Ou seja:

$$
\left.\frac{\partial \dot{\alpha}_{i}}{\partial \alpha_{i}}\right|_{e^{\ell}}=-\frac{\omega_{\ell}}{a} ; i=1, \ldots, s-1 ; \ell=1, \ldots, s .
$$

Temos, então, para cada equilíbrio $e^{\ell}$, a seguinte matriz jacobiana diagonal (omitimos os zeros):

$$
\left[\begin{array}{cccc}
-\frac{\omega_{\ell}}{a} & & & \\
& -\frac{\omega_{\ell}}{a} & & \\
& & \ddots & \\
& & & -\frac{\omega_{\ell}}{a}
\end{array}\right]
$$

Ora, os autovalores de uma matriz desse tipo são reais repetidos e iguais ao valor comum dos elementos da diagonal principal. Como esse valor real é negativo, os equilíbrios em questão são atratores locais. Provamos, portanto, a última e principal proposição deste trabalho:

Proposição 4. Os equilíbrios de estratégia pura $E E P=\left\{e^{i}: i=1,2, \ldots, s\right\}$ são atratores locais. 


\section{Simulação}

Nesta seção apresentamos uma simulação que nos permite ilustrar a coevolução dos salários reais, dos níveis de emprego associados a cada estratégia e do emprego total.

$\operatorname{Seja}\{K, c, \beta\}=\{1,10,1 / 2\}$ econsidere apenas três estratégias $\left\{w_{1}, w_{2}, w_{3}\right\}=\{1,11 / 10,12 / 10\}$ e $A=15$ - note que, supondo, por exemplo, a taxa natural de desemprego, $1-\bar{n}$, igual a $5 \%$, o valor máximo de $A$ é 19 . Substituindo $\alpha_{3}$ por $1-\alpha_{1}-\alpha_{2}$, o equilíbrio de curto prazo correspondente às equações (10)-(13) é:

$$
\begin{aligned}
& p=\frac{6 \sqrt{55}}{\sqrt{55+11 \alpha_{1}+5 \alpha_{2}}} ; \quad n_{1}=\frac{99 \alpha_{1}}{110+22 \alpha_{1}+10 \alpha_{2}} ; \\
& n_{2}=\frac{450 \alpha_{2}}{650+121 \alpha_{1}+55 \alpha_{2}} ; n_{3}=\frac{275\left(1-\alpha_{1}-\alpha_{2}\right)}{8\left(55+11 \alpha_{1}+5 \alpha_{2}\right)} .
\end{aligned}
$$

Com três estratégias, o sistema dinâmico (26) comporta apenas duas equações diferenciais independentes. Escolhemos trabalhar com as equações $\dot{\alpha}_{i}=\left(\alpha_{i} / a\right) \sum_{j=1}^{s} \alpha_{j} \phi^{i, j} ; \quad i=1,2$. Normalizamos $a=1$ e identificamos os seguintes equilíbrios do médio prazo e os autovalores correspondentes:

\begin{tabular}{cccccc}
\hline$\alpha_{1}$ & $\alpha_{2}$ & $\alpha_{3}$ & $\lambda_{1}$ & $\lambda_{2}$ & Tipo de Equilíbrio \\
\hline 1 & 0 & 0 & -0.1369 & -0.1369 & Estável (foco) \\
0 & 1 & 0 & -0.1306 & -0.1306 & Estável (foco) \\
0 & 0 & 1 & -0.125 & -0.125 & Estável (foco) \\
0 & 0.4988 & 0.5012 & -0.0950 & 0.0488 & Instável (sela) \\
0.5051 & 0 & 0.4949 & -0.1004 & 0.0471 & Instável (sela) \\
0.5061 & 0.4937 & 0 & -1044 & 0.0459 & Instável (sela) \\
0.3257 & 0.3323 & 0.3419 & 0.0482 & 0.0446 & Instável (nó) \\
\hline
\end{tabular}

Nas três primeiras linhas da tabela, temos os equilíbrios de estratégia pura; ${ }^{15}$ nas três seguintes, constam os equilíbrios de estratégia mista nos casos em que sobre-

15 Observe que, nestes casos, $\lambda_{1}=\lambda_{2}$, como era esperado à luz dos argumentos da seção precedente. 
vivem apenas duas estratégias; na última linha temos o equilíbrio de estratégia mista com três estratégias. Os equilíbrios de estratégia pura são atratores locais. Os equilíbrios de estratégia mista são instáveis, sendo que, quando sobrevivem apenas duas estratégias, temos instabilidade de sela e o ramo divergente é exatamente a trajetória que liga esses equilíbrios aos de estratégia pura, como podemos constatar no diagrama de fases da Figura 2, obtido por simulação.

Nessa mesma figura podemos identificar claramente as três bacias de atração. Por último, estabelecemos como condição inicial $\left(\alpha_{1}, \alpha_{2}\right)=(0.35,0.29)$. Ela se situa na bacia de atração do equilíbrio keynesiano $(0,0)$, que corresponde ao maior salário nominal, $w_{3}$. As evoluções dos salários reais esperados de cada estratégia, do número de trabalhadores e de firmas de cada setor são apresentadas nas Figuras 3 a 5.

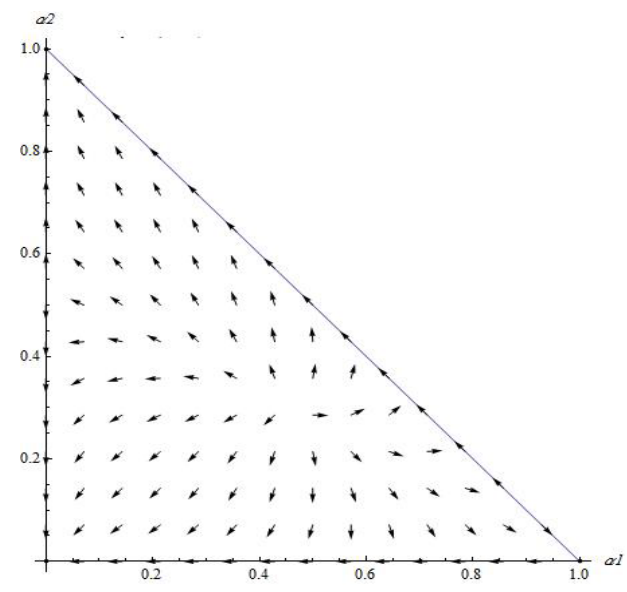

Figura 2 - Diagrama de Fases

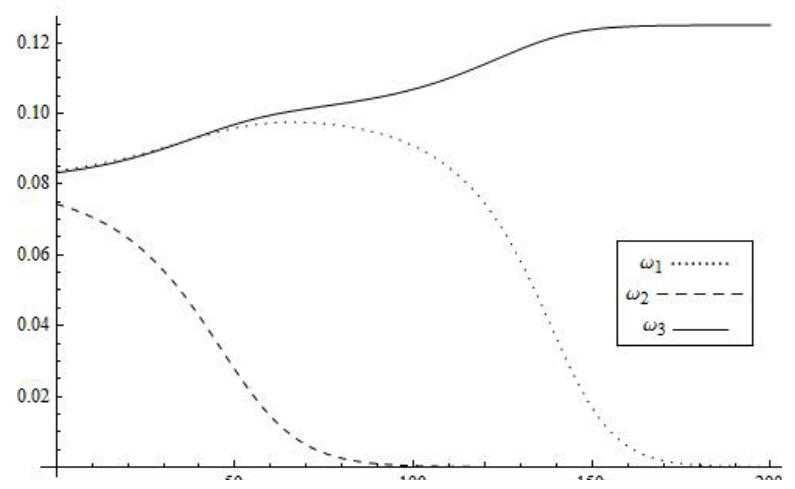

Figura 3 - Evolução dos Salários Esperados 


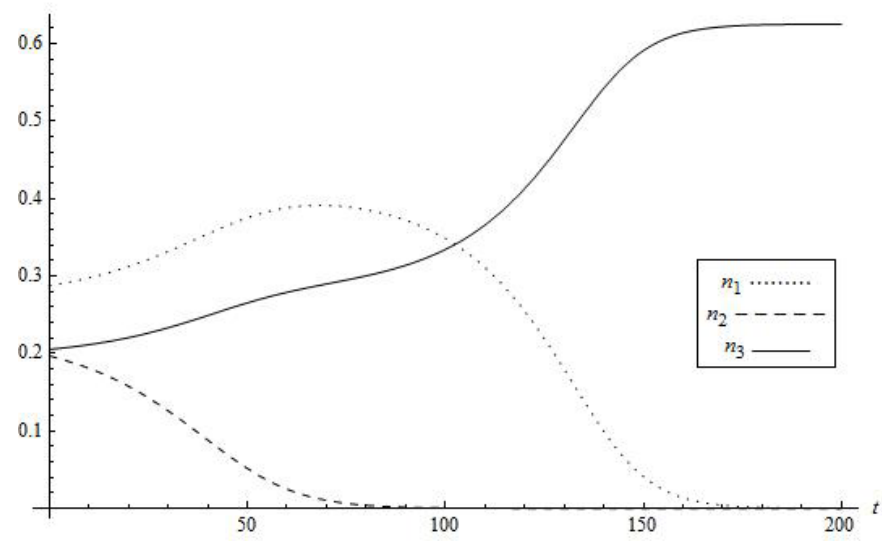

Figura 4 - Evolução do Número de Trabalhadores

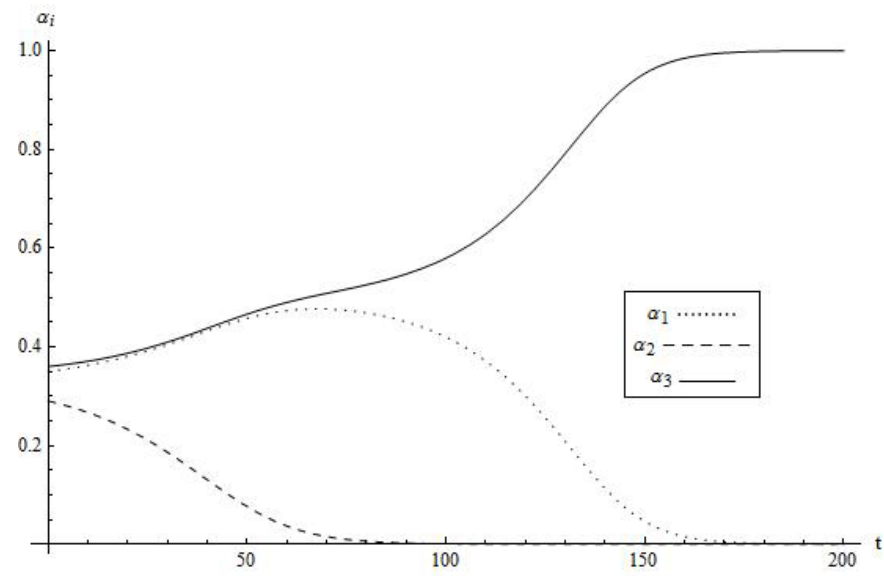

Figura 5 - Evolução do Número de Firmas

A segunda estratégia $w_{2}$ declina continuamente. Trabalhadores e firmas migram para as estratégias $w_{1}$ e $w_{3}$. Por algum tempo, o setor clássico apresenta um nível de emprego superior ao do setor $w_{3}$. Porém, esse setor keynesiano acaba por prevalecer.

O comportamento do emprego total e o do nível de preços são apresentados nas Figuras 6 e 7. O emprego aumenta um pouco nos primeiros períodos, em decorrência do crescimento do setor clássico, mas, em seguida, declina acentuadamente, quanto passa a prevalecer o setor 3. Enquanto existe migração para o setor clássico, 
o emprego total aumenta e o nível de preços se reduz, ambos ligeiramente. À medida que a estratégia $w_{3}$ se impõe e aumentam os custos nominais, o emprego cai e o preço eleva-se transitoriamente até se estabilizarem no equilíbrio final.

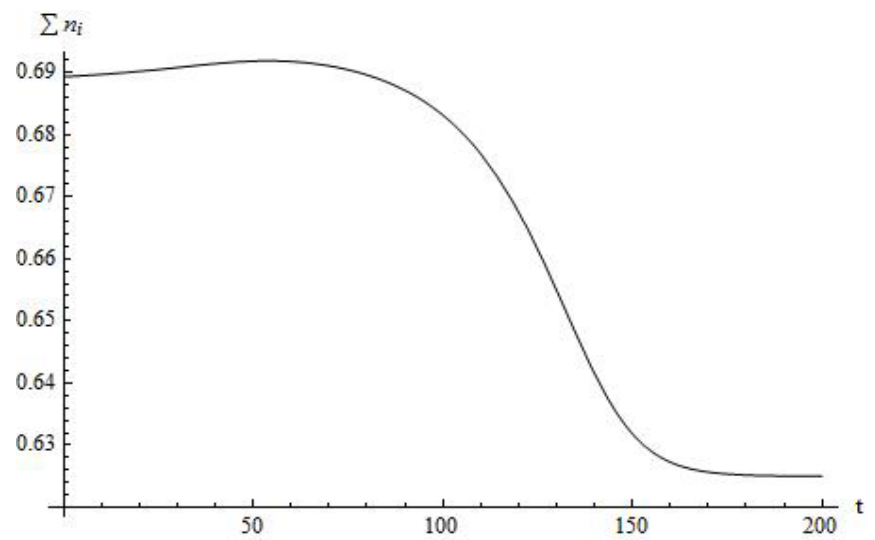

Figura 6 - Evolução do Emprego

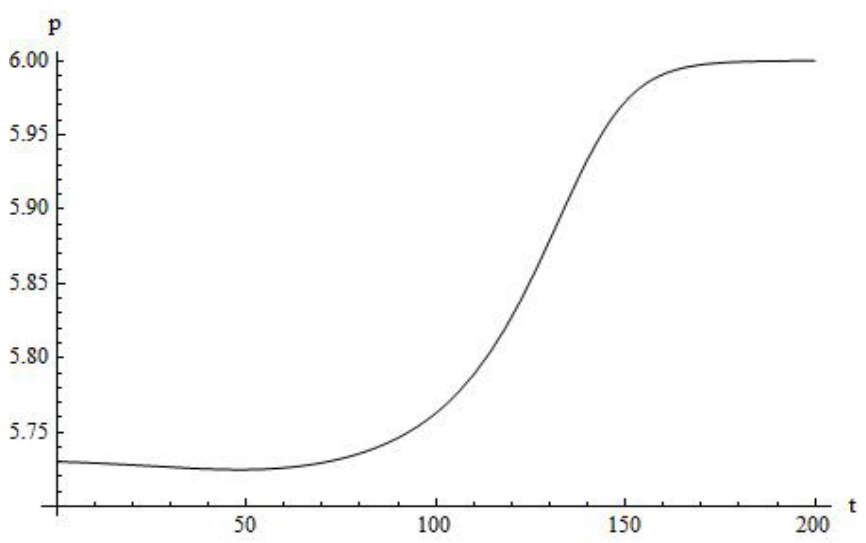

Figura 7 - Evolução do Preço

\section{Conclusão}

Conceber a variação dos salários nominais como um processo evolucionário, no qual os trabalhadores procuram a melhor estratégia, implica, à luz do modelo apresen- 
tado neste artigo, que a economia não tende necessariamente ao pleno emprego. Com efeito, todas as estratégias puras são atratores locais: o pleno emprego é apenas uma entre muitas possibilidades. Esse resultado condiz com a tese fundamental de Keynes que, no primeiro capítulo da Teoria Geral, considerou a situação clássica como limite das situações de equilíbrio possíveis. No entanto, a tese keynesiana foi contestada sistematicamente nas décadas que se seguiram à publicação da Teoria Geral. Como sintetizou Harry Johnson (1961: 12), na macroeconomia keynesiana o desemprego passou a ser concebido como uma situação de desequilíbrio no qual o processo de ajuste ocorria lentamente.

No modelo apresentado neste artigo obtivemos um resultado diferente do tradicional. Mesmo em um contexto dinâmico, a flexibilização dos salários não leva necessariamente à plena ocupação da mão-de-obra. Evidentemente, o resultado foi obtido supondo-se racionalidade imperfeita e um específico processo dinâmico de seleção evolucionária. A nosso ver, no entanto, esse processo é mais plausível do que o retratado pelas equações tradicionais de ajuste do salário nominal ao excesso de demanda, cuja microfundamentação é, como destacamos na introdução, apoiados em Koopmans, no mínimo questionável.

Por último, cabe fazer uma ressalva. O leitor terá observado que não apresentamos a análise das propriedades dinâmicas dos equilíbrios de estratégia mista. Em todas as simulações, esses equilíbrios foram instáveis, mas não foi possível obter esse resultado analiticamente. Não obstante, isso é de certo modo irrelevante para o propósito do artigo. De fato, se existissem equilíbrios de estratégia mista estáveis, isso apenas reforçaria o argumento de que o pleno emprego não é o resultado final e inequívoco do processo dinâmico de flexibilização dos salários.

\section{Referências}

FLASCHE, P.; REINER, F.; SEMMLER, W. Dynamic macroeconomics. Cambridge: The MIT Press, 1997.

HAHN, F.; SOLOW, R. A critical essay on modern macroeconomic theory. Cambridge: The MIT Press, 1997.

HILDENBRAND, W.; KIRMAN, A. P. Equilibrium analysis. Amsterdam: NorthHolland, 1988.

JOHNSON, H. H. The general theory after twenty-five years, American Economic Review: papers and proceedings, vol. LI, n. 2, 1961, p.1-17.

KEYNES, J. M. The general theory of employment, interest and money. London: Macmillan; C.W. VII, 1936. 
KOOPMANS, T. C. Three essays on the state of economic science. McGraw Hill, 1957.

LIPSCHUTZ, S. Schaum's outline of theory and problems of general topology. New York: Schaum Publishing Co, 1965.

NACHBAR, J. H. Evolutionary selection dynamics in games: convergence and limit properties, International Journal of Game Theory, 19, 1990, p. 59-89.

SARGENT, T. J. (1987) Macroeconomic theory. Segunda Edição. New York: Academic Press, 1987.

SOROMENHO, J. E. C.; SILVEIRA, J. J. Demanda e desemprego no médio prazo. Estudos Econômicos, v. 38 n. 4, 2008, p. 747-788.

TOBIN, J. Keynesian models of recession and depression. American Economic Review, 65,1975 , p. 195-202.

TOBIN, J. Price flexibility and the stability of full-employment equilibrium. In: BARKAI, H.; FISCHER, S.; LIVIATAN, N. (Ed.). Monetary theory and thought: essays in honour of Don Patinkin, p. 55-71. London: Macmillan, 1993.

TOBIN, J. Price flexibility and output stability: an old keynesian view. In: SEMMLER, W. Business Cycles: theory and empirical methods, cap. VI. Boston: Kluwer Academic Publisher, 1994. 